\title{
Effects of Nickel leaching from orthodontic appliances in the oral cavity: A review
}

\author{
*Swathi. $\mathrm{R}^{1}$ \\ ${ }^{I}$ IV year BDS, Saveetha Dental college and hospitals, Chennai -77, India
}

\begin{abstract}
Nickel (Ni)-containing alloys are present in a substantial number and wide range of appliances, auxiliaries, and utilities used in orthodontics and thus become an integral part of almost every routine orthodontic intervention. Orthodontic brackets, stainless steel archwires, stainless steel appliances, Nickel titanium wires are some of majorly used the Ni-containing alloy applications. Recent evidence has attributed carcinogenic, mutagenic, cytotoxic, and allergenic actions to $\mathrm{Ni}$ in various forms and compounds. This poses a threat to patients who are potentially allergic to Nickel. So, it is essential to bring into light the use of Nickel free substitutes.
\end{abstract}

Key words: Nickel leaching, orthodontics, allergy, hypersensitivity

\section{Introduction}

Nickel containing alloys are present in a substantial amount and in wide range of appliances and auxiliaries and utilities used in orthodontics and are hence an integral part of every orthodontic intervention. Allergy to nickel is commonly seen in the population and is a concern for orthodontists, who might be required to treat patients with allergy. Nickel titanium (Ni-Ti) wires contain about $47-55 \%$ of nickel $^{1}$. It is also a component of orthodontic brackets, stainless steel, etc. Recent evidence has attributed carcinogenic ${ }^{2}$, mutagenic $^{3}$, cytotoxic ${ }^{3}$ and allergenic action ${ }^{5-7}$ to nickel in various forms and compounds, posing a threat to patients undergoing orthodontic treatment who are potentially allergic to nickel. Nickel leaches from orthodontic appliances and forms a solution in saliva and causes the effects. ${ }^{8}$

\section{Immune Reaction And Other Biologic Reactions}

The allergenic response to nickel is a type IV delayed hypersensitivity reaction. The reaction is induced by sensitized T-cells (Tdth, Th1, Th2, Tc) whichon contact with specific antigen releases cytokines that produce biological effects on macrophages, leucocytes and tissue cells ${ }^{9}$. Hence, pre-sensitized individuals develop contact dermatitis or mucositis. Nickel leaching from orthodontic appliances can result in mucositis which develops over a period of do 3 days to up to 3 weeks. Solution of $\mathrm{Ni}(0.05 \mathrm{~mol} / \mathrm{L})$ has been found to impair phagocytosis of bacteria by human polymorhonuclear leukocytes in vitro. Nickel ions may affect the chemotaxis of leukocytes, stimulate the neutrophils to become aspherical causing them to move slowly and inhibit calcium dependent contractile activity by depolarizing the neutrophil cell membrane. There is an abundance of evidence supporting the carcinogenic and mutagenic actions of $\mathrm{Ni}$ in cell cultures.

\section{Epidemiology}

Nickel allergy is more common in women than in men. $11 \%$ of all women and $20 \%$ of women between the age of 16 and 35 have a sensitivity to nickel. $2 \%$ of men are sensitive to nickel. This is caused due to contact of nickel from jewelry. Hypersensitivity reactions to nickel in orthodontic patients has not been reported much. Occurrence of harmful response is only $0.1-0.2 \%$. Nickel leaching of orthodontic bands, brackets and stainless steel or NiTiarchwires has been shown to occur in vitro within the first week and declines thereafter. This coincides with the approximate time frame of delayed hypersensitivity reactions.

\section{Pre-Disposing Factors}

Saliva and other intra oral conditions like food, oral hygiene products and fluoride may potentially corrode the nickel in the alloy and release it onto the oral mucosa. Factors including intra-oral temperature, $\mathrm{pH}$, salivary composition, duration of exposure wear of the wire due to friction from sliding mechanics, abrasion, presence of solder, strain of the wire and most importantly the amount of nickel leached are the factors determining the allergenic potential of nickel present from a particular appliance. Other predisposing factors include genetics and the presence of major histocompatibility complex haplotype Nickel sensitivity has also been found in asthmatic patients. 


\section{Nickel Hypersensitivity In Orthodontic Patients And Its Diagnosis}

The issue of hypersensitivity reactions in orthodontic patients has been reviewed during the last 20years. Majority of evidence on this adverse effect associated with conventional orthodontic appliances has been derived from scarce case reports. These reports have indicated that insertion of NiTi wire alloys may Occasionally lead to formation of rashes, swellings and painful erythematous lesions in the oral mucosa. The diagnosis of a response to nickel in the oral mucosa is more difficult than on the skin. The patient will mention regarding a known allergy to nickel during the verbal medical history review. The patient should be pre-warned about the possible allergic reactions after receiving the orthodontic appliances. Diagnosis of nickel allergy can be confirmed by cutaneous sensitivity test (patch testing) using $5 \%$ nickel sulphate in petroleum jelly ${ }^{12}$.Signs and symptoms include- burning sensation, gingival hyperplasia, labial desquamation, angular chelitis, erythema multiforme, periodontitis, stomatitis with mild to severe erythema, papular perioral rash, metallic taste or loss if taste, numbness, soreness at side of the tongue. Extra oral manifestations of nickel allergy can be of intra oral origin. The nickel leachability test consists of $1 \%$ dimethylglyoxime and $10 \%$ ammonium hydroxide which are mixed just prior to use. A positive test for nickel leaching is a colour change to red. The symptoms have to be managed individually and the NiTi wire should me removed and be replaced with stainless steel. Orthodontic appliances with nickel free substitutes should be considered. ${ }^{13}$

\section{Discussion}

Several case studies have shown the diagnosis of nickel contact hypersensitivity was supported by the onset of symptoms after placing NiTiarchwires mainly. ${ }^{13} \mathrm{~A}$ potential sensitisation mechanism is the use of nickel jewellery. This explains the frequent incidence of nickel hypersensitivity in females than in males. An atopic history maybe a significant predisposing factor. ${ }^{13}$

\section{Conclusion}

The diagnosis of nickel contact hypersensitivity was supported by the onset of symptoms after placing NiTiarchwires mainly. A potential sensitisation mechanism is the use of nickel jewellery. This explains the frequent incidence of nickel hypersensitivity in females than in males. An atopic history maybe a significant predisposing factor. ${ }^{13}$

\section{Reference}

[1]. Brantley, W. A. Orthodontic wires. In: Brantley WA, Eliades T, eds. Orthodontic Materials: Scientific and Clinical Aspects.Stuttgart, Germany: Thieme; 2000:78-100

[2]. Lewis, C. G. and F. W. Sunderman . Metal carcinogenesis in total joint arthroplasty. Animal models. ClinOrthop 1996.329:(suppl). S264-S268.

[3]. Kasprzak, K. S. and K. Bialkowski . Inhibition of antimutagenic enzymes, 8-oxo-dGTpases, by carcinogenic metals. J InorgBiochem 2000.79:231-236.

[4]. Pereira, M. L. , A. Silva , R. Tracana , and G. S. Carvalho . Toxic effects caused by stainless steel corrosion products on mouse seminiferous cells. Cytobios 1994. 77:73-80.

[5]. Veien, N. K. , E. Bochhorst , T. Hattel , and G. Laurberg . Stomatitis or systemically-induced contact-dermatitis. Contact Dermatitis 1994. 30:210-213

[6]. Al-Waheidi, E. M. Allergic reaction to nickel orthodontic wire: a case report. Quintessence Int 1995. 26:385-387

[7]. Al-Waheidi, E. M. Allergic reaction to nickel orthodontic wire: a case report. Quintessence Int 1995. 26:385-387

[8]. In Vivo Aging of Orthodontic Alloys: Implications for Corrosion Potential, Nickel Release, and Biocompatibility

[9]. DELAYED HYPERSENSITIVITY-TYPE GRANULOMA FORMATION AND DERMAL REACTION INDUCED AND ELICITED BY A SOLUBLE FACTOR ISOLATED FROM SCHISTOSOMA MANSONI EGGS

[10]. Stenman E, Bergman M. Hypersensitivity reactions to dental materials in a referred group of patients. Scand J Dent Res 1989;97:76-83.

[11]. Oshida, Y. , R. C. L. Sachdeva , and S. Miyazaki . Microanalytical characterization and surface modification of TiNi orthodontic archwires. Bio-Medical Materials and Engineering 1992. 2:51-69.

[12]. In vitro Lymphocyte Proliferation as Compared to Patch Test Using Gold, Palladium and Nickel

[13]. Nickel allergy and orthodontics, a review and report of two cases ... 